\title{
Lack of clinically relevant differences between combination therapy and monotherapy in COPD
}

To the Editor:

BATEMAN et al. [1] reported significantly greater mean improvements with indacaterol/glycopyrronium combination therapy, Ultibro Breezhaler (QVA149) compared to monotherapy with either indacaterol $(0.07 \mathrm{~L})$, glycopyrronium $(0.09 \mathrm{~L})$ or tiotropium $(0.08 \mathrm{~L})$ for the primary outcome of trough forced expiratory volume in $1 \mathrm{~s}(\mathrm{FEV} 1)$ at week 26 in patients with chronic obstructive pulmonary disease (COPD). However, such improvements were all less than the $0.1-0.14 \mathrm{~L}$ range that represents the minimal clinically important difference (MCID) for FEV1 [2]. Pointedly, the mean differences between combination therapy and monotherapy for the secondary outcomes of dyspnoea (transition dyspnoea index score) and health status (St. George's Respiratory Questionnaire score) were also less than the respective MCID values, with a significant difference between Ultibro Breezhaler (QVA149) and tiotropium at week 26. Analysis of individual responders revealed a significantly higher proportion of patients exceeding the MCID for transition dyspnoea index ( $\geqslant 1$ unit) and St. George's Respiratory Questionnaire ( $\geqslant 4$ units) with QVA149 versus tiotropium but not indacaterol or glycopyrronium. These data do not therefore support the conclusion that QVA149 demonstrated clinically relevant superiority versus their respective monotherapy components in COPD.

@ERSpublications

Clinically relevant differences not found between combination and monotherapy http://ow.ly/thbE4

Brian J. Lipworth

Asthma and Allergy Research Group, Division of Cardiovascular and Diabetes Medicine, University of Dundee, Ninewells Hospital and Medical School, Dundee, UK.

Correspondence: B.J. Lipworth, Asthma and Allergy Research Group, Division of Cardiovascular and Diabetes Medicine, University of Dundee, Ninewells Hospital and Medical School, Dundee, DD1 9SY, UK.

E-mail: b.j.lipworth@dundee.ac.uk

Received: Aug 152013 | Accepted: Aug 252013

Conflict of interest: Disclosures can be found alongside the online version of this article at www.erj.ersjournals.com

\section{References}

Bateman ED, Ferguson GT, Barnes N, et al. Dual bronchodilation with QVA149 versus single bronchodilator therapy: the SHINE study. Eur Respir J 2013; 42: 1484-1494.

2 Cazzola M, MacNee W, Martinez FJ, et al. Outcomes for COPD pharmacological trials: from lung function to biomarkers. Eur Respir J 2008; 31: 416-469.

Eur Respir J 2014; 43: 1204 | DOI: 10.1183/09031936.00143513 | Copyright @eERS 2014

From the authors:

We would like to thank B.J. Lipworth for his letter and interest in the SHINE study. B.J. Lipworth incorrectly describes the conclusion of the SHINE study article. Nowhere in the article is it stated that QVA149 "demonstrated clinically relevant superiority versus its respective monotherapy components in COPD”. Rather, we concluded that "dual bronchodilation with once-daily QVA149 demonstrated superior and clinically meaningful outcomes versus placebo and superiority versus treatment with a single bronchodilator" [1]. The conclusion on QVA149 versus placebo is justified by improvements in trough forced expiratory volume in $1 \mathrm{~s}$ (FEV1) being twice the accepted minimal clinically important difference (MCID) of $100 \mathrm{~mL}$ [2] and that for the transition dyspnoea index (TDI) exceeding the MCID of $>1$ unit improvement [3]. The improvement in the St George's Respiratory Questionnaire (SGRQ) total score (least squares mean difference versus placebo -3.99 units at week 12) fell just short of the MCID of 4 units reduction [4]. 
Further, we acknowledged in the discussion section of the article, that a limitation of the study is the "difficulty in evaluating the clinical significance of spirometric and other clinical end-points (e.g. TDI and SGRQ total scores) versus active (i.e. monocomponent) treatments" [1]. Current standards for MCID thresholds were proposed for comparisons versus placebo or for improvements versus baseline, but have not been confirmed to be applicable or attainable in comparisons either between active comparators, or between combination products and their monotherapy components [2].

Based on this, and as recommended in the new European Medicines Agency's Committee for Medicinal Products for Human Use guidance [5], a predefined clinically relevant mean treatment difference of $0.06 \mathrm{~L}$ for trough FEV1 was set in the SHINE study protocol for QVA149 monotherapy comparisons. This is consistent with published data comparing free long-acting $\beta_{2}$-agonist (LABA)+long-acting muscarinic antagonist (LAMA) combinations with individual LABA or LAMA therapies [6, 7]. Along with the statistical significance seen for QVA149 versus all monotherapies, the observed treatment differences for trough FEV1 also met, and exceeded, the predefined clinically relevant threshold of $0.06 \mathrm{~L}$ (QVA149 versus indacaterol, $0.07 \mathrm{~L}$, glycopyrronium, $0.09 \mathrm{~L}$, and tiotropium, $0.08 \mathrm{~L}$ ). It is also important to note that the MCID is defined as the smallest difference that patients can perceive (at the individual level) as beneficial. The focus should not only be on mean differences between treatments but, therefore, also on the number and percentage of patients who experience relevant improvement (i.e. responder analyses). Responder analyses carried out for this study also corroborate the clinical relevance of these data. A significantly higher proportion of patients on QVA149 demonstrated an improvement of $>100 \mathrm{~mL}$ in trough FEV1 from baseline $(64.3 \%)$ than all the monotherapies (indacaterol $46.2 \%$, glycopyrronium $43.2 \%$ and tiotropium $46.6 \%)$ and placebo $18.9 \%$ ( $\mathrm{p}<0.001$ for all treatment comparisons).

Because no published consensus on MCID thresholds between active comparators exists, we did not predefine clinically relevant differences in TDI or SGRQ total scores when comparing QVA149 with its monotherapy components or tiotropium. The reduction in SGRQ total score in the placebo group was unusually high after 26 weeks of treatment $(>7$ units, two to three times the placebo effect seen in most clinical trials), which may have diminished the opportunity to observe MCID differences between QVA149 and active comparators. Despite this, QVA149 demonstrated the greatest mean reduction in SGRQ total score out of all the active treatments tested; $64 \%$ of patients achieved an improvement of $\geqslant 4$ units and $>50 \%$ an improvement of $\geqslant 8$ units, compared with baseline values. Furthermore, a greater proportion of patients achieved the MCID in TDI focal score of $>1$ unit with QVA149 (68.1\%), compared with indacaterol (64.6\%), glycopyrronium (63.7\%), tiotropium $(59.2 \%)$ and placebo $(57.5 \%)$. Therefore, we believe that our original summary is both accurate and justified.

○ @ERSpublications

Dual bronchodilation with once-daily QVA149 provides superior treatment outcomes in COPD versus single bronchodilators http://ow.ly/sne7T

Eric Bateman on behalf of the SHINE study report authors

Dept of Medicine, University of Cape Town, Cape Town, South Africa.

Correspondence: E. Bateman, Division of Pulmonology, Dept of Medicine, University of Cape Town, George Street, Mowbray 7700, Cape Town, South Africa. E-mail: eric.bateman@uct.ac.za

Received: Sept 062013 | Accepted: Sept 252013

Conflict of interest: Disclosures can be found alongside the online version of this article at www.erj.ersjournals.com

\section{References}

1 Bateman ED, Ferguson GT, Barnes N, et al. Dual bronchodilation with QVA149 versus single bronchodilator therapy: the SHINE study. Eur Respir J 2013; 42: 1484-1494.

Donohue JF. Minimal clinically important differences in COPD lung function. COPD 2005; 2: 111-124.

3 Witek TJ Jr, Mahler DA. Minimal important difference of the transition dyspnoea index in a multinational clinical trial. Eur Respir J 2003; 21: 267-272.

4 Jones PW. Interpreting thresholds for a clinically significant change in health status in asthma and COPD. Eur Respir J 2002; 19: 398-404.

5 European Medicines Agency. Guidelines on clinical investigation of medicinal products in the treatment of chronic obstructive pulmonary disease (COPD). London, European Medicines Agency, 2012.

6 Wang J, Jin D, Zuo P, et al. Comparison of tiotropium plus formoterol to tiotropium alone in stable chronic obstructive pulmonary disease: a meta-analysis. Respirology 2011; 16: 350-358.

7 Mahler DA, D'Urzo A, Peckitt C, et al. Combining once-daily bronchodilators in COPD: indacaterol plus tiotropium versus tiotropium alone. Am J Respir Crit Care Med 2011; 183: 1591. 